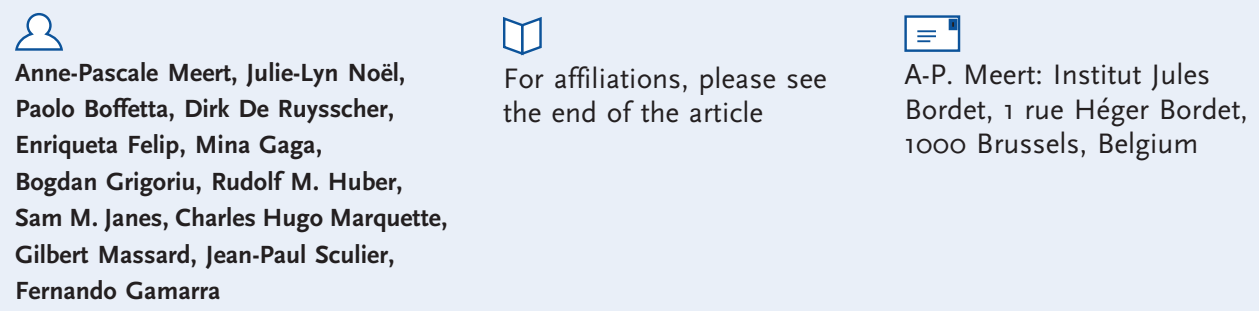

\section{[1]}

For affiliations, please see the end of the article

\section{(2)}

ap.meert@bordet.be

\title{
Thoracic Oncology HERMES: a European syllabus towards a harmonised education and training of Thoracic Oncology specialists
}

\section{Introduction}

From a medical perspective, the unification of Europe has resulted in an increasing number of physicians crossing borders and practising in different countries. In order to ensure and maintain a high quality of healthcare across Europe, the European Union (EU) has demonstrated its intent to achieve harmonisation of medical education and training. The EU implemented recognition of diplomas and certificates of qualification in medicine in all member countries through a European Parliament directive on the recognition of professional qualifications issued in 2005 [1]. The goal is to achieve high-quality patient care across Europe. At postgraduate level and later career stages, this will only be possible if knowledge and skills levels are more uniform. This holds true for thoracic oncology, which is a growing discipline. Lung cancer is currently the deadliest malignant disease worldwide. Malignant pleural mesothelioma is the most frequent and deadly occupational cancer. However, there have been important recent advances in our understanding of thoracic tumour biology, resulting in better imaging and sampling techniques, better tumour tissue characterisation and the development of promising new therapies.

The European Respiratory Society (ERS) has recognised these developments and has created the thoracic oncology assembly. The "Thoracic Oncology Action Plan" has been published in the ERJ [2]. The plan includes forming a Task Force for a European initiative for quality management in lung cancer care, the development of a network with other assemblies and an educational programme with the aim of improving the training of thoracic oncology specialists. Thoracic oncology is an expanding subspecialty that requires the participation of surgeons, radiation oncologists, respiratory physicians, oncologists, pathologists and radiologists among others. Collaboration between different specialists is essential in the management of thoracic tumours. However, training in thoracic oncology is not well defined in the various European countries and depends on the subject-specific background of the physicians who want to become thoracic oncology specialists. The professional career of thoracic oncology specialists also differs across countries. Pharmacotherapy of lung cancer is for example mainly prescribed by respiratory
Statement of Interest $J$-L. Noël is an employee of ERS; P. Boffetta reports various grants from $\mathrm{NIH}$ and other agencies, and personal fees from consultancy, outside the submitted work; SM Janes has received payment for lectures from Roche (advisory committee), travel/ accommodation/meeting expenses, unrelated to activities listed, and for travel to the world lung cancer conference 2013 from Roche; F. Gamarra reports grants from the ERS, during the conduct of the study. 
medicine specialists in some European countries but by oncologists in others.

The ERS felt comprehensive and multidisciplinary educational standards for thoracic oncology were required. This is consistent with the ERS mission of alleviating the suffering of patients with oncologic respiratory disease. Several organisations, including the ERS, currently offer different courses to educate thoracic oncology specialists. It is however recognised that because of the recent advances in thoracic oncology and the need for an interdisciplinary approach, a consensus-based syllabus and curriculum are required. The development of such documents would be an important contribution to the harmonisation of thoracic oncology training and practice and support free movement of specialists within Europe while increasing standardised expertise.

According to a survey completed in May 2012 regarding training in and the practice of thoracic oncology, the overall opinion of the respondents was that there is a need in Europe for standardised training. To address this, the ERS supported a project within the framework of the HERMES (Harmonised Education in Respiratory Medicine for European Specialists) initiative. Thus, the Thoracic Oncology HERMES project was launched to develop training standards, relevant educational activities and supporting educational resources.

\section{Objectives}

The Task Force began with a comprehensive review of the current status of thoracic oncology training and certification in Europe including recommendations made by other European or international societies. Using this information, the Task Force aimed to develop a comprehensive European Thoracic Oncology core syllabus, which was consensus-based and

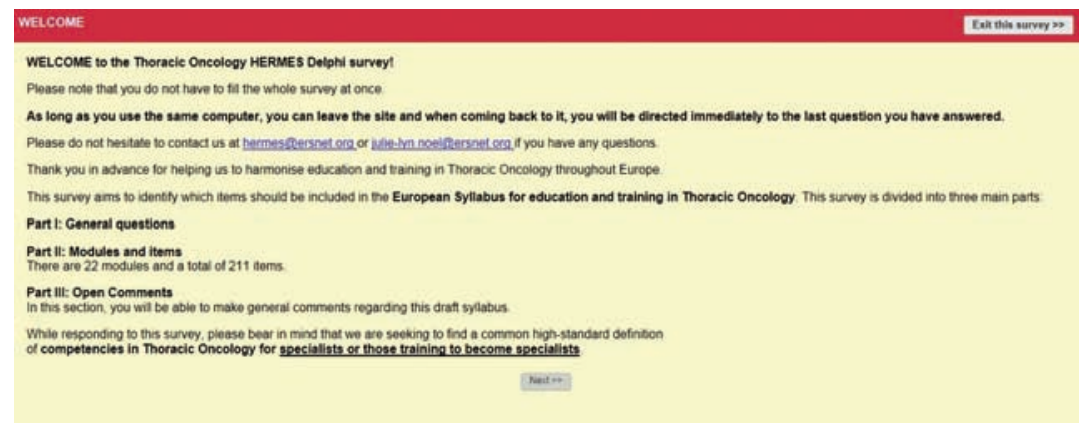

Figure 1

Thoracic Oncology Online Survey using the SurveyMonkey platform. included the main disciplines working in the field. Syllabus items were discussed keeping in mind the aim of raising the thoracic oncology competency level across Europe. The results of the discussion were used to set priorities within the syllabus.

\section{Methodology}

The first objective of the Task Force has been to obtain a comprehensive overview of the current thoracic oncology training and certification programmes in Europe. Representatives of the Forum of European Respiratory Societies (FERS) were contacted. Each respiratory society was asked to provide a national respondent who would be able to support the Task Force and provide information on thoracic oncology training and organisation of practice in his/her respective country. The Task Force also sought the collaboration of the European Initiative for Quality Management in Lung Cancer Care (EIQMLCC-ERS Task Force) that provided a list of thoracic oncology specialists from almost all European countries and the rest of the world. The FERS-selected national respondents and the EIQMLCC-ERS Task Force national representatives were approached and asked to provide information by completing an online standardised questionnaire (www.surveymonkey.com). Further information was searched on the internet and in medical databases, such as PubMed. Using the information about training in different countries, the Task Force prepared a proposal for a syllabus. The proposal was designed with careful consideration that it would be fit for the use of physicians from different specialties and backgrounds who wanted to train in thoracic oncology.

In a second step, a modified Delphi process to generate consensus with respect to the syllabus items was performed through an online survey. This modified Delphi process is similar to that used for the other HERMES task forces.

The syllabus was developed in accordance with the HERMES methodology with supporting ERS staff. The participants in the Delphi process included an expert group and public group. The expert group consisted of the members of the Task Force, the national respondents elected by FERS and the national representatives of the EIQMLCC-ERS Task 
Force. The public group of respondents consisted of members of the ERS Thoracic Oncology Assembly 11 who, upon joining the ERS, chose to affiliate themselves because of interest in the topic.

To ensure a wide-ranging consultation throughout the Delphi process, the following criteria were stipulated:

- The following subspecialties should be represented: respiratory physicians, thoracic surgeons, medical oncologists, radiation oncology;

- All European regions should be represented: Central, Eastern, Northern, Southern and Western.

The HERMES project methodology was adopted in order to achieve consensus-based educational standards based on wide consultation. The processes adopted were considered to strengthen the specialty with the involvement of representatives who are stakeholders in the development of training in thoracic oncology. The whole process allowed a large number of participants to be surveyed and consulted. This ensured that participants developed a feeling of ownership over the outcome, which is essential for later integration of the study output into practice [3-6].

The composition of the Task Force reflects the interdisciplinary character of thoracic oncology. Together with respiratory physicians, a medical oncologist (E. Felip), a thoracic surgeon (G. Massard), and a radiotherapist (P. de Ruysscher) were invited to participate. They are active members of ESMO (European Society of Medical Oncology), ESTS (European Society of Thoracic Surgery) and ESTRO (European Society of Radiation Oncology) respectively.

\section{Delphi round I}

In compliance with the standardised steps used for Delphi studies, participants in the first Delphi round received a proposal for the syllabus drafted by the Task Force. They were asked to approve the items and suggest further items that should be included and considered.

\section{Delphi round 2}

In a second round, participants received a questionnaire where they were asked to give a desired level of competence to each item previously identified by the group and to determine which part of the syllabus needed further discussion or amendment. Their responses were then collated.

\section{Review round}

Representatives of the different medical disciplines and countries reviewed the draft of the syllabus. They commented individually on whether the syllabus adequately represented all the disciplines and was acceptable according to the organisation of training and practice in the different countries.

\section{Plenary meeting}

The plenary meeting was a round-table discussion of the Task Force members considering the results of the survey and feedback of all respondents. Controversies and issues were discussed and served as a takeoff point for improving the whole syllabus. This meeting was crucial in identifying challenges and opportunities as well as for careful consideration of next steps.

The ERS staff in Lausanne provided support for all of the steps (J-L. Noël: collection of information, contacts with national respondents, collation of results, statistical aggregation in Delphi round, organisation of meetings, minutes-taking, etc.).

Facilitated group discussion techniques are used for each face-to-face meeting with the ERS staff and the medical education advisor of the ERS (J. Busari). This follows on from the methods used for delivering competency-based education standards in the HERMES initiative and has been adapted for various projects: Adult Respiratory Medicine [7], Paediatric Respiratory Medicine [8], Spirometry [9], Respiratory Sleep Medicine [10], Respiratory Critical Care Medicine [11], and Respiratory Physiotherapy, which is currently ongoing.

\section{Results}

The Thoracic Oncology Task Force was officially launched during the ERS Annual Congress in September 2011 in Amsterdam. Between October 2011 and March 2012, the Task Force started with a survey with the aim of assessing the current practice and organisation of thoracic oncology in Europe. The 
questionnaire was sent to an "expert group", consisting of the Task Force members, national representatives from the EIQMLC which included 37 European and seven nonEuropean countries and the national representatives of the FERS in which 35 countries are represented. Furthermore, all members of the Thoracic Oncology Assembly of the ERS were invited to participate in the "public group". $80 \%$ of the participants responded that management of thoracic oncology conditions by a multidisciplinary team (MDT) is mandatory or recommended in their countries. However, only $29 \%$ stated that thoracic oncology is a recognised specialty or subspecialty. Thoracic oncology training seems to differ between countries, with many of them requiring rotations in both respiratory and oncology units.

In March 2012, the Task Force met to create the initial draft syllabus to be used for the Delphi 1 process. Task Force members used the HERMES syllabus in adult respiratory medicine as background information [7]. The first draft of the syllabus was discussed at a plenary meeting defining the modules. The items to be included in each module were formulated by working groups. Responsible persons were assigned for each module with the following tasks that were to be accomplished:

- Assess if module is complete or covering all the topics that need to be included for thoracic oncology.

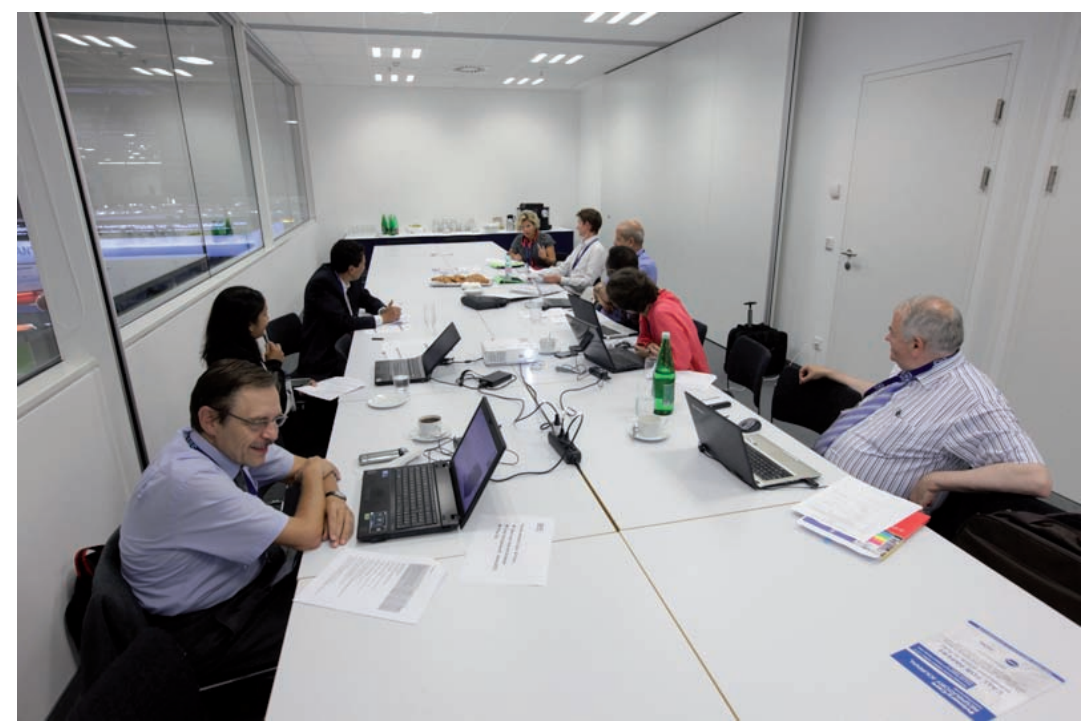

Figure 2

Thoracic Oncology HERMES Task Force discussion in September 2012, Vienna.
- Complete the module with additional items.

- Delete items which are not needed.

- Indicate whether it is an optional or mandatory module.

- Determine pre-requisite items in the respective fields in the multidisciplinary team of thoracic oncology.

Further review of the draft from the Task Force, especially by the members representing other societies (ESMO, ESTRO, ESTS) was performed.

A first Delphi survey was then sent to the "experts" and "public" groups as defined previously in July 2012. They had to vote on each module of the syllabus indicating if it should be "mandatory" or "optional". Expert and public respondents also approved each of the syllabus items by indicating "agree" or "disagree", gave general feedback on each module and suggested further items or changes in the composition in the modules.

In September 2012, the results of Delphi 1 were discussed. An approval rate of $<80 \%$ of the respondents was considered controversial and 15 items were identified in this category. In the setting of facilitated group discussion, each item was discussed, and improved by rephrasing or deleting from the syllabus. All general comments were also discussed and used to improve the whole syllabus. Further electronic exchange was performed to improve the second draft ready for Delphi 2 .

In December 2012, respondents for Delphi 2 were asked to mark each item and assign a level of competence. Being multidisciplinary, the Thoracic Oncology HERMES project was faced with the challenge to come up with generic competencies common to the various disciplines involved in the daily practice of thoracic oncology. These generic competencies must be specified in order to be fit to run a thoracic oncology service whether you are a respiratory specialist, thoracic surgeon, medical oncologist, radiotherapist or pathologist.

The next step was to define "levels of competence" for each item of the syllabus. After several discussions and taking into account the efforts of the HERMES leadership to unify the definitions of "levels of competence" in the different HERMES projects, the Task Force agreed to use the following definitions:

Level 1: foundation competency to be used in daily practice by everybody involved in 
the field. It is a requisite to function in the multidisciplinary team/setting.

Level 2: intermediate competency to be demonstrated or applied with consultation within the multidisciplinary team.

Level 3: specialised competency which has the highest level of complexity and specialisation.

In March 2013, Delphi 2 results were discussed in a face-to-face plenary meeting. This time, the answers were not as clear as in the first survey with 44 non-consensual items. There were many discrepancies between the participants of the "expert" and "public" groups and many answers diverged from the suggestions of the Task Force members. For many items the level of competence were decided by a narrow majority of votes. Changes were made during the plenary workshop. Finally, in view of the discrepancies and difficulties encountered, the Task Force decided that in this first edition, the levels of competence grading for the syllabus items will not be published.

The final syllabus consists of 23 modules and 207 items (see appendix). Table 1 shows the modules of the syllabus.

\section{Discussion}

It did not prove possible to include representatives of all countries or regions, and the Task Force and FERS-selected National Respondents represent 14 countries. One of the challenges encountered in arriving at a consensus relates to national differences of acceptability and applicability of the syllabus items recommended. In the case of Thoracic Oncology, acceptability issues may relate to cultural differences in terms of who gives or takes charge of the actual care. There is also the difficulty of accepting the syllabus item recommendation as this means an accompanying change to the already existing structures or the non-existent structures in their local contexts. Applicability relates to the limited resources locally so that the recommendations are difficult to deliver. Balancing of applicability and acceptability for each recommendation was carefully considered by the experts involved. The project relied heavily on the informed contribution of the involved experts in finding an acceptable and applicable recommendation.

The method of reaching a broader consensus through a Delphi process has the advantage of including the opinion of many participants from different countries, with a wide variety of backgrounds. A disadvantage of this process is not all respondents have the same level of expertise. This may be less the case with the volunteers of the Thoracic Oncology Assembly, but also here the local background in the management of thoracic malignancies and the speciality of the respondent are relevant.

A main challenge was to account for the different backgrounds of the potential thoracic oncology diploma candidates. A medical oncologist does not need to achieve the same level of performance in interventional bronchoscopy as a respiratory physician and we cannot expect a radiotherapist to perform a lobectomy himself.

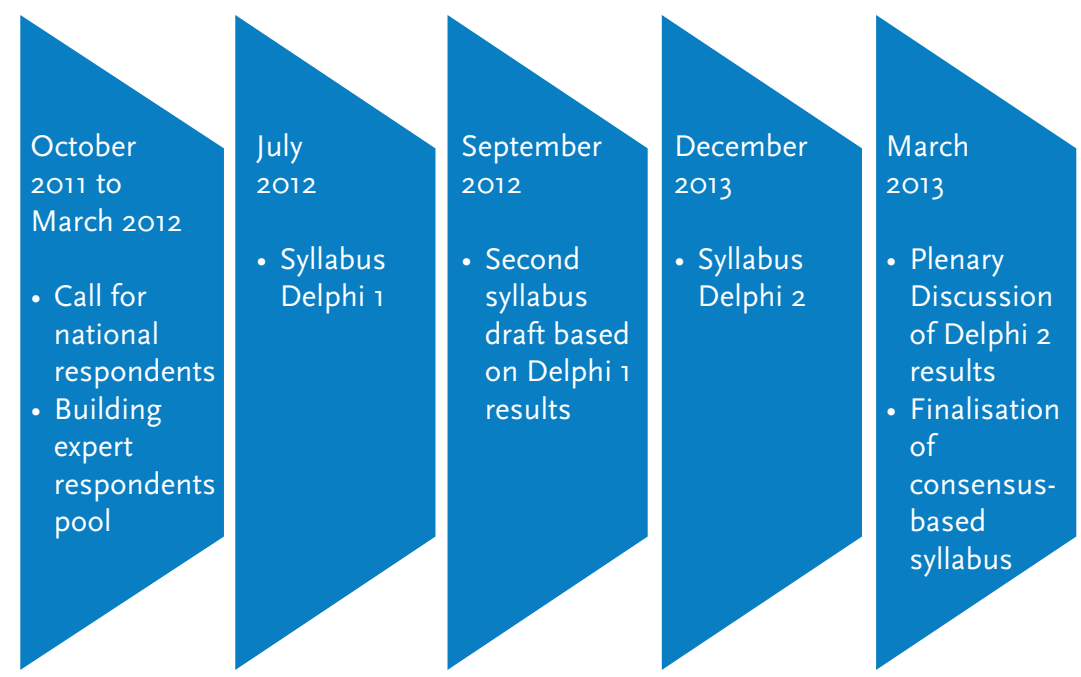

Figure 3

Timelines of the Delphi process in producing the consensus-based syllabus. 
Table 1. Thoracic Oncology HERMES Syllabus Modules

\begin{tabular}{ll}
\hline A & General principles of the biology of thoracic cancers \\
\hline B & Aetiology and epidemiology \\
\hline C & Clinical presentations \\
\hline D & Diagnostic procedures \\
\hline E & Imaging \\
\hline
\end{tabular}

\begin{tabular}{ll}
\hline F & $\begin{array}{l}\text { Diagnostic and interventional bronchoscopic techniques and medical } \\
\text { thoracoscopy }\end{array}$ \\
\hline G & Clinical and pathological staging \\
\hline $\mathbf{H}$ & Pathology of intrathoracic tumours \\
\hline $\mathbf{I}$ & Prognostic factors / predictive markers \\
\hline $\mathbf{J}$ & Principles of thoracic surgery \\
\hline $\mathbf{K}$ & Management of surgical complications \\
\hline $\mathbf{L}$ & Principles of radiation therapy \\
\hline $\mathbf{M}$ & Principles of systemic therapy \\
\hline $\mathbf{N}$ & Side-effects of systemic therapy and their management \\
\hline $\mathbf{O}$ & Combined modality treatments \\
\hline $\mathbf{P}$ & Management of particular groups of patients \\
\hline $\mathbf{Q}$ & Treatment evaluation and follow-up \\
\hline $\mathbf{R}$ & Supportive care \\
\hline $\mathbf{S}$ & Methodologies for clinical practice and research \\
\hline $\mathbf{T}$ & Ethics \\
\hline $\mathbf{U}$ & Cancer-related immunology \\
\hline
\end{tabular}

This challenge also relates to the difficulties of assigning levels of competence at the syllabus phase.

The aim of grading the syllabus items was to differentiate between topics that need to be mastered by every thoracic oncology specialist (for example knowing a patient should be offered a lobectomy) and those that are very complex (for example techniques in performing a lobectomy and treating specific complications) and therefore only intended for a certain group.

The first Delphi survey on the list of modules and items reached easy consensus. The second one had conflicting results.
The syllabus is basically a list of topics which serves as groundwork in building the curriculum and examination. As noted, the Task Force has decided not to publish the levels of competence grading for the syllabus items at the present time because of the difficulties encountered. For the creation of the curriculum, which is the next step, the knowledge, skills, attitudes and behaviour associated with each syllabus item will be defined. Additionally we will give recommendations for the training and evaluation of the skills, specifying the level of performance that the candidates need to achieve.

It was also felt that the modules are oriented to the medical specialties rather than surgical and that there are maybe more details in the "oncology" modules. This is probably due to the leading role of the ERS (and therefore probably greater orientation toward respiratory physicians). A discussion ensued on doing separated tracks for the different disciplines. Discussion currently focuses on pre-requisite items for each of the disciplines involved in thoracic oncology which will be further detailed in the curriculum.

Trainee involvement was also a discussion point. According to the state of affairs survey done in May 2012, specialised centres exist in at least five countries. In France, 25\% of trainees in pulmonology are practising thoracic oncology or have a special interest. The group decided that the national respondents will be asked to provide a list of trainees if available.

A national training programme survey is envisaged to be done as a follow up to the initial state of affairs survey in thoracic oncology. Information gathered will be useful for the local implementation of the outputs of this project. This points to the applicability of the syllabus in different countries with consideration of the roles of respiratory physicians and oncologists.

\section{Conclusion}

The consensus-based syllabus is a first step in achieving harmonised training in thoracic oncology. It is the first European syllabus of Thoracic Oncology that includes the main treating disciplines. In our opinion, this is essential to provide patients with comprehensive, high-quality care. As a next step, curriculum recommendations will be developed. The 
curriculum will effect the syllabus with stated competencies or skills. Further explicit recommendations concerning the training and assessment of would-be thoracic oncology specialists will need to be defined. These steps will then be the groundwork for future development of a certification and accreditation program. The aim of harmonised training through these educational standards and activities will hopefully eventually lead to the ultimate goal of the very best of care for all lung cancer patients wherever they live in Europe.

\section{Affiliations}

A-P. Meert and J-P. Sculier: Service des Soins Intensifs et Urgences Oncologiques, Oncologie Thoracique, Institut Jules Bordet (ULB), Brussels, Belgium; J-L. Noël: Educational Activities, European Respiratory Society Headquarters, Lausanne, Switzerland; P. Boffetta: The Tisch Cancer Institute and Institute for Translational Epidemiology, Mount Sinai School of Medicine, NY, USA; D.
De Ruysscher: Radiation Oncology, University Hospitals Leuven/ KU Leuven, Leuven, Belgium; E. Felip: Thoracic Oncology Unit, Oncology Department, Vall d'Hebron University Hospital, Vall d'Hebron Institute of Oncology (VHIO), Barcelona, Spain; M: Gaga: $7^{\text {th }}$ Respiratory Medicine Dept, Athens Chest Hospital "Sotiria", Athens, Greece; B. Grigoriu: Regional Institute of Oncology, University of Medicine and Pharmacy, lasi, Romania; RM Huber: Division of Respiratory Medicine and Thoracic Oncology - Campus Innenstadt, University of Munich and Thoracic Oncology Centre Munich, Ludwig-Maximilians Universität, Munich, Germany; SM. Janes Lungs for Living Research Centre, University College London, UK; $\mathrm{CH}$. Marquette: Institute for Research on Cancer and Ageing in Nice IRCAN, INSERM U1081CNRS, Nice, France; G. Massard: Service de Chirurgie Thoracique, Hôpitaux Universitaires de Strasbourg, Strasbourg, France; and F. Gamarra: Sektion Pneumologie Innenstadt und Thorakale Onkologie, Medizinische Klinik und Poliklinik 5, Ludwig-Maximilians University, Munich, Germany.

\section{Reference}

1. Directive 2005/36/EC of the European Parliament and of the Council of 7 September 2005 on the recognition of professional qualifications Official Journal of the European Union 2005255.

2. Sculier JP, Vansteenkiste J, Schonfeld N, et al. Thoracic oncology in Europe: the ERS action plan by the Thoracic Oncology Assembly. Eur Respir J 2010; 36: 1227-1228.

3. Keeney $\mathrm{S}$, McKenna $\mathrm{H}$. Research guidelines for the Delphi survey technique. J Adv Nurs 2000; 32: 1008-1015.

4. Goodman CM. The Delphi technique: a critique.J Adv Nurs 1987; 12: 729-734

5. Powell C. The Delphi technique: myths and realities. J Adv Nurs 2003; 41: 376-382.

6. Walker AM, Selfe J. The Delphi method: a useful tool for the allied health researcher. BrJ Ther Rehabil 1996; 3: 677-681.

7. Loddenkemper R, Séverin T, Eiselé JL, et al. HERMES: a European core syllabus in respiratory medicine. Breathe 2006; 3: 59-70.
8. Gappa M, Noël J-L, Severin T, et al. Paediatric HERMES: a European syllabus in paediatric respiratory medicine. Breathe 2009; 5: 237-241.

9. Steenbruggen, S. Mitchell, T Séverin, et al., Spirometry HERMES: a European training programme and qualification in spirometry practice. Breathe 2011; 7: 259-275.

10. De Backer W, Simonds A, Horn V, et al. Sleep HERMES: a European Core Syllabus in Respiratory Disorders during sleep. Breathe 2011; 8: 61-68.

11. Artigas A, Vassilakopoulos T, Brochard L, et al. Respiratory Critical Care HERMES: a European core syllabus in respiratory critical care medicine. Breathe 2012; 8: 217-229.

12. Artigas A, Pelosi P, Dellweg D, et al. Respiratory critical care HERMES syllabus: defining competencies for respiratory doctors. Eur Respir J 2012; 39: 1294-1297. 


\section{Thoracic Oncology HERMES Syllabus}

\section{A: General principles of the biology of thoracic cancers}

1 Carcinogenesis

2 Tumour immunology

3 DNA damage and repair

4 Epigenetics

5 Oncogenes and tumour suppressor genes

6 Cell cycle and apoptosis

7 Growth factors and signalling pathways

8 Oncogenic driver mutations

9 Tumour microenvironment and angiogenesis

10 Mechanisms involved in metastasis

\section{B: Aetiology and epidemiology}

1 Descriptive epidemiology of lung and other thoracic cancers

2 Tobacco

3 Air pollution (outdoor and indoor)

4 Asbestos

5 Other occupational carcinogens

6 Radon decay products

7 Chronic inflammation (COPD, infections)

8 Genetic susceptibility and gene-environment interactions

\section{C: Clinical presentations}

1 Performance status

2 Signs and symptoms

2.1 Cough

2.2 Haemoptysis

2.3 Chest pain

2.4 Dyspnoea

2.5 Wheezing

2.6 Stridor

2.7 Hoarseness

2.8 Phrenic nerve paralysis

2.9 Dysphagia

2.10 Pleural effusion

2.11 Palpable peripheral lymph nodes

2.12 Superior vena cava syndrome

2.13 Pancoast syndrome

2.14 Pericardial effusion

2.15 Lymphangitic spread

2.16 Bone metastases

2.17 Brain metastases

2.18 Liver and adrenal metastases

2.19 Other metastatic sites

3 Paraneoplasic syndromes

3.1 Cachexia

3.2 Hypercalcaemia

3.3 Thromboses and pulmonary embolism

3.4 Syndrome of inappropriate antidiuretic hormone

3.5 Ectopic adrenocorticotropin hormone syndrome

3.6 Other paraneoplastic syndromes 
Thoracic Oncology HERMES Syllabus

\section{D: Diagnostic Procedures}

1 Principles of screening, target population, biases

2 Imaging (Chest radiograph, Computed tomography, PET and PET-CT, Ultrasound, Bone scaning, Magnetic resonance imaging)

3 Imaging guided biopsy

4 Bronchoscopy

5 Medical thoracoscopy

6 Open lung biopsies and VATS

7 Mediastinoscopy

8 Pulmonary nodule: definition and diagnostic algorithm

9 Tumour markers

10 Diagnosis and staging algorithms

11 Fitness for therapy

\section{E: Imaging}

1 Methods and indications of thoracic cancer and other thoracic tumours

1.1 Chest radiographs

1.2 Computed tomography

1.3 Bone scanning

1.4 PET and PET-CT

1.5 Others (MRI sonography)

2 Interpretation of imaging patterns of thoracic tumours

3 Solitary pulmonary nodule

3.1 Imaging, Bayesian approach and diagnostic algorithms for solitary nodules

\section{F: Diagnostic and interventional bronchoscopic techniques and medical thoracoscopy}

1 Indications and contraindications of bronchoscopy and pleuroscopy

2 Therapeutic endoscopy (stents, cryotherapy, laser techniques, brachytherapy)

3 Therapeutic pleuroscopy

\section{G: Clinical and pathological staging}

1 TNM description

$2 \mathrm{~T}$ description

$3 \mathrm{~N}$ description

$4 \mathrm{M}$ description

5 Stage grouping

\section{$\mathrm{H}$ : Pathology of intrathoracic tumours}

1 Conditioning of cytological and biopsy specimens for pathology/molecular analysis

2 Preinvasive lesions for lung neoplasms

3 Pathology of lung neoplasms

4 Pathological classification and molecular pathology of nonsmall cell lung cancer

5 Pathology and molecular pathology of neuroendocrine and small cell lung cancer

6 Pathology and molecular pathology of mesothelioma

7 Thymoma and germ cell tumors ("frequent" mediastinal tumours)

8 Rare mediastinal tumors

9 Interpretation of pathological reports

\section{I: Prognostic factors/predictive markers}

1 General methodological considerations

2 Clinical factors

3 Histopathological factors

4 Molecular markers 


\section{Thoracic Oncology HERMES Syllabus}

\section{J: Principles of thoracic surgery}

1 Oncologic principles of surgery for lung tumours

2 Indications, contraindications and perioperative care for thoracic surgery

3 Surgical techniques

4 Alternatives to lobectomy and pneumonectomy

5 Surgery in the context of multi-modality treatment

6 Palliative surgical procedures

7 Surgery for synchronous and metachronous cancers

8 Surgery for oligo-metastatic lung cancer

9 Diagnostic, curative and palliative surgery for mediastinal tumours

10 Curative and palliative surgery of malignant mesothelioma

11 Pulmonary metastases from other sites and its management

\section{K: Management of surgical complications}

1 Pain control after thoracic operations

2 Post-operative pneumonia: diagnosis, treatment and prevention

3 Empyema and bronchial fistula after pneumonectomy/lobectomy

4 Prolonged airleak

5 Post-operative bleeding

6 Acute respiratory distress syndrome (ARDS)

7 Cardiac complications after surgery: infarction, arrhythmia

8 Chronic pain after thoracotomy

9 Chronic complications after pneumonectomy

10 Other chronic complications

\section{L: Principles of radiation therapy}

1 Radiotherapy planning and techniques

2 Indications and contraindications for radiotherapy

3 Thoracic radiotherapy with curative intent for lung cancer

4 Thoracic palliative radiotherapy

5 Prophylactic, therapeutic and palliative cranial irradiation

6 Stereotactic ablative radiosurgery

7 Palliative radiotherapy for other metastatic sites

8 Radiotherapy for mesothelioma and thymoma

9 Management of side-effects

\section{M: Principles of systemic therapy}

1 Basis of cytotoxic therapy

2 Basis of biological therapy

3 Indications and contraindications for systemic therapy including chemotherapy and targeted agents

4 NSCLC (Nonsmall cell lung carcinoma)

4.1 First-line induction and maintenance therapy for advanced NSCLC including chemotherapy and targeted agents

4.2 Second line therapy for NSCLC

4.3 Further lines of treatment

5 Small cell lung cancer (SCLC)

5.1 First-line therapy for SCLC

5.2 Second line therapy for SCLC

5.3 Further lines of treatment

6 Systemic therapy of mesothelioma

7 Systemic therapy for mediastinal tumours 


\section{$\mathrm{N}$ : Side effects of systemic therapy and their management}

1 Quantification of side-effects

2 Side-effects of chemotherapy

2.1 Haematological side-effects

2.1.1 Neutropenia and febrile neutropenia

2.1.2 Anaemia

2.1.3 Thrombopenia

2.2 Mucositis/oesophagitis

2.3 Alopecia and dermatological toxicity

2.4 Nausea and vomiting

2.5 Diarrhoea

2.6 Neurotoxicity

2.7 Ototoxicity

2.8 Hepatic toxicity

2.9 Nephrotoxicity

2.10 Cardiovascular toxicity

2.11 Extravasation

2.12 Infertility and teratogenesis

2.13 Electrolyte imbalance

3 Side-effects of molecular therapy

3.1 Dermatological toxicity

3.2 Diarrhoea

3.3 Hepatic toxicity

3.4 Cardiovascular toxicity

3.5 Pulmonary toxicity

3.6 Nephrotoxicity

\section{O: Combined modality treatments}

1 Adjuvant chemotherapy for early NSCLC

2 Neo-adjuvant chemotherapy for early NSCLC

3 Combined radio-chemotherapy for locally advanced NSCLC

4 Combined radio-chemotherapy for limited SCLC

5 Combined treatment for mesothelioma

\section{P: Management of particular groups of patients}

1 Elderly

2 Poor performance status patient

3 Unfit patients due to comorbidities

4 Patients with HIV

5 Preneoplastic and preinvasive lesions

6 Synchronous, metachronous lung tumours

7 Oligometastatic disease

8 Patients with brain metastases

9 Ischaemic heart disease in potentially operable patients

9.1 Screening and work up

9.2 Treatment options and recommendations

10 Atherosclerosis of carotid vessels in the surgical patient

11 Strategy in patients with combined lung and extra-thoracic malignancies (head and neck, bladder, colon, kidney, breast, etc.)

\section{Q: Treatment evaluation and follow-up}

1 Survival/progression-free survival

2 Response assessment

3 Other surrogate endpoints

4 Quality of life/symptom improvement

5 Follow-up 


\section{Thoracic Oncology HERMES Syllabus}

\section{R: Supportive care}

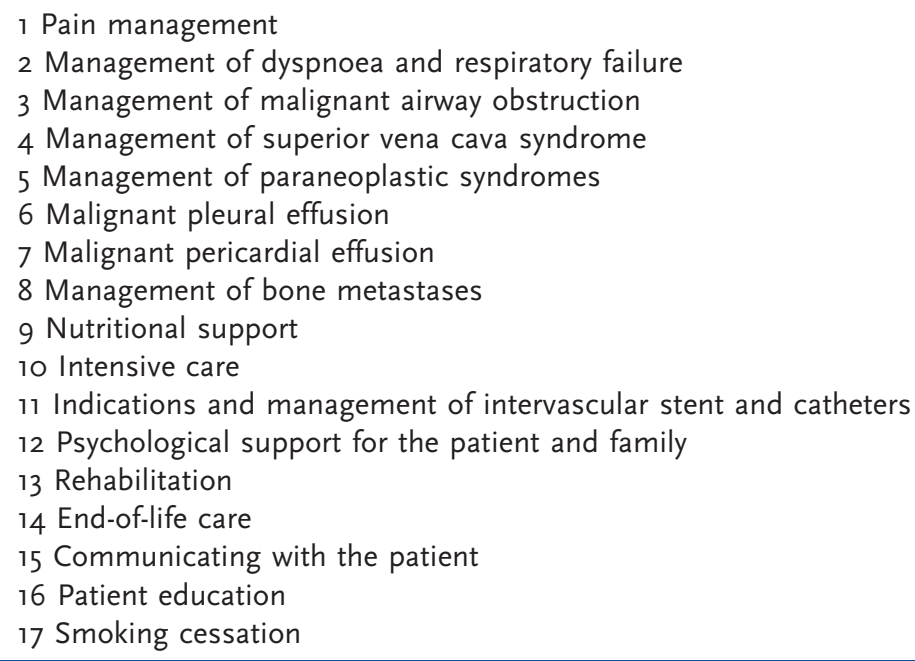

\section{S: Methodologies for clinical practice and research}

1 Study design and phases

2 Principles of biobanking

3 Statistical analysis

4 Evidence based decision

5 Guidelines assessment and application

\section{T: Ethics}

1 Ethical issues

2 Conflicts of interest

\section{U: Cancer-related immunology}

1 Basic principles of cancer-related immunology

2 Specific immunotherapy

\section{V: Economic considerations in lung cancer treatment}

1 Principles of quality management in thoracic malignancies

2 Quality-adjusted life-year (QALY)

3 Principles of cost-effectiveness of chemotherapy, targeted therapy, surgical therapy and radiotherapy

4 Cancer registries 